\title{
Halal and kosher slaughter methods and meat quality: a review
}

\begin{abstract}
There are many slaughter procedures that religions and cultures use around the world. The two that are commercially relevant are the halal and kosher methods practiced by Muslims and Jews respectively. The global trade in red meat and poultry produced using these two methods is substantial, thus the importance of the quality of the meat produced using the methods. Halal and kosher slaughter per se should not affect meat quality more than their industrial equivalents, however, some of their associated pre- and post-slaughter processes do. For instance, the slow decline in blood pressure following a halal pre-slaughter head-only stun and neck cut causes blood splash (ecchymosis) in a range of muscles and organs of slaughtered livestock. Other quality concerns include bruising, hemorrhages, skin discoloration and broken bones particularly in poultry. In addition to these conventional quality issues, the ñspiritual qualityò of the meat can also be affected when the halal and kosher religious requirements are not fully met during the slaughter process. The nature, causes, importance and mitigations of these and other quality issues related to halal and kosher slaughtering and meat production using these methods are the subjects of this review.
\end{abstract}

Keyword: Ritual slaughter; Nutrition; Stunning; Safety; Meat spiritual quality 